\title{
Mathematical Modeling of Water Profile surrounding the root surface in Soil, in Cortex Tissues and in Xylem Tubes while Root Uptake the Water from the Soil *
}

\author{
Avhale.P.S ${ }^{1}$ Kiwne S.B ${ }^{2}$ \\ ${ }^{1}$ Department of Mathematics \\ Shivaji Arts and Science College Kannad \\ Dist.Aurangabad (MH), India, \\ e-mail:-avhaleps@yahoo.com \\ ${ }^{2}$ Head of Department of Mathematics \\ Deogiri College Aurangabad(MH), India \\ e-mail:-sbkiwne@yahoo.com
}

July 5,2014

Subject classification (AMS):- 35XX-35Kxx , 76XX-76Rxx, 76XX-7605, 76XX-76Zxx-76Z05 and 92XX-92Bxx.

\begin{abstract}
The root system of plant uptake water and nutrient from the soil. The present paper explain systematic description of mathematical expression for water profile surrounding the root surface in porous soil, in root surface, i.e, in cortex tissues, and in stem, i.e., in xylem tune while root uptake water from the soil. We use basic principles of physics and fluid-dynamics of water flow. Tiny xylem tubes as water transport channel in the stem, root surface work as semipermeable membrane and soil is considered as porous medium. We resolve mathematical model of water profile using variety of boundary conditions by analytically. As new approach, we obtain water profile surrounding the root surface by using constant internal pressure in the root as boundary condition and we solve radial diffusion equation by using Goodman integral transformation and separation of variables.
\end{abstract}

Key words and Phrases: Plant root, water uptake by root, water profile, soil water pressure, water pressure in xylem and water pressure at inner radius of root.

\section{Introduction}

The primary physiological function of root is uptaking the water as well as nutrients and transport to leaves for photosynthesis. Investigations and observation of the uptake of water and nutrient in plant root and stem can be traced back to many years ago, it possesses importance in point of view of agricultural production and economical development. Plant uptake water and nutrient simultaneously. In traditional farming like planting and agricultural the mechanism of water and nutrients is invaluable for utilizing water and fertilizer for increasing production. Now a new trend of planting inedible plant emerge on industrial basis. The view of planting inedible plant are prevent the salinization, desertification of soil, to clean pollution of heavy metals, radioelement and plant's mining. To collect the valuable metals, like gold, in soil by planting some plants whose roots possess a special capability of absorbing the valuable metals. The plant of genus Bauhina have many species out of which Bauhinia variegata plant extract is analysed and found it contain microparticles of gold [18]. Since ancient times Bauhinia racemosa Lam. family: Caesalpinaceae has been an integral part of life in India. Leaves of Bauhinia racemosa are traditionally used on occasion of Dashera festival as symbol of gold in India [5]. Recently proved 
that Bauhina racemosa extract also contain micro particles of gold.

Water enters into root through root surface from the soil.Then it flow through micro-tube of xylem in the stem to rich leaves and being vaporized into air from the pores on leaves. The transport of water in plant can affect the water flow in soil, and vice versa. Then study of Mathematical model for plant water uptake divides into the parts: (1) the flow of water in the porous soil (2) water flow into root through root surface (3) flow of water through micro-tube of xylem (4) vaporization of water through leaves.

In recent years, a number of researchers from various fields, such as physics, applied mathematics and plant physiology, paid more attention to develop mathematical model for water and nutrient uptake. The outstanding work in this field is done by T.Roose and proposed a mathematical model for uptake of water and nutrient. Roose work is development of Nye, Tinker and Barber model for water and nutrient uptake assuming that the root is an infinitely long cylinder [4][13]. Before this model many root water uptake model like, a linear root water uptake model ( Prasad, Rama 1988 ) [11], CERES ( Ritche, 1985) [12] and SWAP (Van Dam et al, 1997) [17] was developed. In point of view of above discussion it important to develop mathematical model of water profile in porous soil, in root surface and in xylem for further study of ability for survival of plant in different soils. Also it is important to obtain solutions for differential model of water profile in different situation.

\section{Flow of water in porous soil}

In 1856 Henry Darcy make experiment and concluded that, rate of flow (i.e. volume of water per unit time), $Q$ is (a) proportional to the cross-sectional area $A$, (b) proportional to $\left(h_{1}-h_{2}\right)$, the lengths $h_{1}$ and $h_{2}$ are measured with respect to some arbitrary datum level and (c) inversely proportional to the the length $L$ [1].

$$
Q=K \frac{A\left(h_{1}-h_{2}\right)}{L} .
$$

Where $K$ is a coefficient of proportionality which is known as soil hydraulic conductivity. Equation (2.1) is known as Darcy formula. Specific discharge, $\mathbf{u}$ as the volume of water flowing per unit time through a unit cross-sectional area normal to the direction of flow, i.e., $\mathbf{u}=\frac{Q}{A}$, we obtain

$$
\mathbf{u}=K J .
$$

Where $J=\frac{\left(h_{1}-h_{2}\right)}{L}$ in one dimension. In three dimension the generalization of equation (2.2) is given by

$$
\mathbf{u}=K J=-K \text { grand } \phi=-\nabla \phi .
$$

The average areal porosity is equal to the volumetric porosity $n$, the portion of the area $A$ available to flow is $n A$. The average velocity $V$ of flow is given by

$$
V=\frac{Q}{n A}=\frac{\mathbf{u}}{n} .
$$

Another form of Darcy formula is in term of specific discharge, i.e., Darcy flux is written as

$$
\mathbf{u}=-\frac{k}{\mu}(\operatorname{grand} p-\rho \mathbf{g}) .
$$

Where $K=\frac{k \rho g}{\mu}, \mu$ is dynamic viscosity of fluid, $\rho=\frac{\gamma}{g}$ is the density of the fluid, $\gamma$ is specific weight of water and $g$ is gravitation, $k$ is the medium's permeability and $\mathbf{g}=-g \mathbf{l} \mathbf{z}$ is the vector gravity acceleration directed downward. If fluid is not compressible then the $\nabla u=0$.

The equation for the conservation of water in the soil with no water sink is given by [13],

$$
\frac{\partial \phi_{l}}{\partial t}+\nabla \cdot \mathbf{u}=0
$$

Where $\mathbf{u}$ is given by,

$$
\mathbf{u}=-\frac{k}{\mu}[\nabla p-\rho g \hat{k}] .
$$

The $k$ soil permeability is just differ from hydraulic conductivity $K$ by scaling factor which is given by,

$$
K=\frac{k \rho g}{\mu}
$$

Where $\rho g$ present the density of water and gravity. If soil is saturated then we have $\rho g \hat{k}=0$ therefore the Darcy flux is given by $\mathbf{u}=-\frac{k}{\mu}[\nabla p]$. However, the conductivity of unsaturated soil is determined from experiments in terms of effective water saturation, where effective water saturation $S$ is defined as

$$
S=\frac{\phi_{l}-\phi_{l, r}}{\phi_{l, s}-\phi_{l, r}}
$$


Where $\phi_{l}$ is the moisture content of the soil, $\phi_{l, r}$ is the residual (minimum) moisture content of the soil and $\phi_{l, s}$ is the saturated moisture content of the soil, i.e. the soil porosity. Van Genuchten drive a formula for the soil hydraulic conductivity as a function of soil moisture content and porosity which is given by [10]

$$
K=K_{s} S^{1 / 2}\left[1-\left(1-S^{1 / m}\right)^{m}\right]^{2} \text { for } 0<m<1 .
$$

Where $K_{s}$ is the hydraulic conductivity of fully saturated soil. In addition to deriving the soil hydraulic conductivity formula (2.10), he also presents a formula for the suction characteristic. He uses the pressure head $h$ suction $-p>0$ can be written in terms of pressure head, i.e., $-p=-\rho g h$. Hence we have

$S=\left[\frac{1}{1+(\alpha|h|)^{n}}\right]^{m}=\left[\frac{1}{1+\left[\frac{\alpha}{\rho g}(-p)\right]^{n}}\right]^{m}, \quad m=1-\frac{1}{n}$,

$$
0<m<1 \text {. }
$$

Where $\alpha$ is a fitting parameter that can be inversely linked to the bubbling pressure.

We write equation (2.6) of conservation of water in the soil in terms of relative moisture content $S$ using the full van Genuchten formulas, then we get the following nonlinear diffusion-convection equation

$$
\left(\phi_{l, s}-\phi_{l, r}\right) \frac{\partial S}{\partial t}=\nabla \cdot(D(S) \nabla S)-\frac{\rho g}{\mu} \frac{d k}{d S} \frac{\partial S}{\partial z} .
$$

Where $D(S)$ is called the soil water diffusivity and it is defined as

$$
\begin{aligned}
D(S)= & \frac{k(S)}{\mu}\left|\frac{\partial p}{\partial S}\right|=\frac{k_{s} \rho g}{\mu} \times \frac{(1-m)}{\alpha m} S^{1 / 2-1 / m} \\
& {\left[\left(1-S^{1 / m}\right)^{-m}+\left(1-S^{1 / m}\right)^{m}-2\right] . }
\end{aligned}
$$

and

$$
\begin{gathered}
\frac{d k(S)}{d S}=k_{s}\left[\frac{1}{2} S^{-1 / 2}\left[1-\left(1-S^{1 / m}\right)^{m}\right]^{2}+2 S^{(-1 / 2+1 / m)}\right. \\
\left.\left[1-\left(1-S^{1 / m}\right)^{m}\right]\left(1-S^{1 / m}\right)^{(m-1)}\right] .
\end{gathered}
$$

Where $k_{s}$ is soil permittivity.

\section{Range of validity}

In the flow through conduits, the Reynolds number, $R_{e}$, which is dimensionless number expressing the ratio of inertial to viscous forces acting on the fluid, is used as a criterion to distinguish between laminar flow occurring at low velocities and turbulent flow occurring at higher velocities. By analogy, a Reynolds number is defined also for flow through porous media [1]

$$
R_{e}=\frac{\mathbf{u} d}{v}
$$

Where $d$ is some representative length of the porous matrix and $v$ is the kinematic viscosity of the fluid. Practically all evidence indicates that Darcy's law is valid as long as the Reynolds number does not exceed some value between 1 and 10 . There the equation (2.12) valued as long as Darcy's law obeys, .i.e., for Reynolds number value less than 1 flow will be at very low pressure gradients through low-permeability sediments, also for Reynolds number value greater than 10 there will be large flows through high permeability gradients.

\section{Water flow through root surface inside the root}

Water flow through root surface, then there will be change in water pressure, which serve as boundary condition in equation of water conservation in the root. The root surface membrane work as semipermeable membrane represents the water movement path across the cortex to the xylem. The rate of water uptake by plant roots per unit surface area $J_{w}\left[\mathrm{~cm}^{3} \mathrm{~cm}^{-2} \mathrm{~s}^{-1}\right]$ is given by [9][13]

$$
J_{w}=k_{r}(\nabla P-\sigma \Delta \Pi) .
$$

Where $k_{r}$ is the speed of water flow through the root surface membrane per unit difference in water pressure on either side of the membrane, $\nabla P$ is the hydraulic water pressure across the membrane, $\sigma$ is the reflection which give relation between osmotic pressure to hydrostatic and $\Delta \Pi$ is the osmotic pressure difference across the membrane.

Neglecting the osmotic pressure effects on water uptake by plant roots and $D$ is the water diffusivity in cortical tissues, then for a root surface membrane with the inner boundary at $a_{x}$ and an outer boundary at the root surface $a$, the water pressure distribution inside this membrane is given by a solution to the equation

$$
\frac{\partial p_{m}}{\partial t}=\frac{D}{r} \frac{\partial}{\partial r}\left(r \frac{\partial p_{m}}{\partial r}\right) .
$$

With boundary conditions given for example by $p_{m}=$ $p$ on $r=a$ and $p_{m}=p_{r}$ at $r=a_{x}$, where $p_{m}$ is the water pressure in the root cortex tissues, $p$ is the water pressure at the root surface and $p_{r}$ is the water pressure in the xylem.

Non-dimensionalising the above equation with typical timescale $[t]$ and root radius $a$, we find that the 
dimensionless problem becomes

$$
\frac{a^{2}}{D[t]} \frac{\partial p_{m}}{\partial t}=\frac{1}{r} \frac{\partial}{\partial r}\left(r \frac{\partial p_{m}}{\partial r}\right) .
$$

With

$$
p_{m}=p \text { on } r=1 \text { and } p_{m}=p_{r} \text { at } r=a_{x} / a=r_{x} \text {. }
$$

For small order radius, water pressure distribution profile is at pseudo steady state, i.e., the time derivative term in the equation (3.3) is small, and the steady state solution is given by [7]

$$
\begin{array}{r}
p_{m}=\frac{p-p_{r}}{-\ln \left(r_{x}\right)} \ln (r)+p \\
\approx \frac{p-p_{r}}{1-r_{x}}(r-1)+p \text { for } r_{x}<r<1 .
\end{array}
$$

Hence, in dimensional terms the flux of water through the unit of root surface area is given approximately by

$$
\left.D \frac{\partial p_{m}}{\partial r}\right|_{r=a}=\frac{D}{a-a_{x}}\left(p-p_{r}\right)
$$

Where $k_{r}=D /\left(a-a_{x}\right)$ is generally known as a root surface membrane hydraulic conductivity. Using boundary condition equation (3.6) at the root surface, we can be written as

$$
\frac{k(S)}{\mu} \frac{\partial p}{\partial r}=k_{r}\left(p-p_{r}\right), \text { at } r=a
$$

Where $k(S)$ is the soil water permeability, $k_{r}$ is the radial conductivity of root surface membrane, $p$ is the water pressure in the soil, $p_{r}$ is the water pressure in the root xylem and $a$ is the radius of the root.

\section{Flow of water in stem from root to leaves through the xylem lon- gitudinally upwards direction}

Interior parts of root is made of macro tubes of xylem with average radius $r_{m}$. Assume that the number of the micro-tubes is $N$. The total area cross-section of the micro tubes can be written as $N \pi r_{m}^{2}=\pi R^{2}$. We assume that the water path effectively by a single tiny circular tube with the radius $R$. Water motion in the interior of root is described by one dimensional Poiseuille flow along the pipe with variable cross section area of radius $r[2][13]$.

$$
\mu \nabla^{2} u=\nabla p-\rho g \hat{\mathbf{k}}
$$

Where $u$ is water velocity profile with non-slip boundary condition which is given by

$$
u(r, z)=\frac{r^{2}-R^{2}}{4 \mu}\left[\frac{\partial p_{r}}{\partial z}-\rho g\right] .
$$

The total flux of water passing upwards through the cross section at the level $z$ is given by

$$
q=-\frac{\pi R^{4}}{8 \mu}\left[\frac{\partial p_{r}}{\partial z}-\rho g\right] .
$$

Let $k_{x}=\frac{\pi R^{4}}{8 \mu}$, then equation (4.3) becomes

$$
q=-k_{x}\left[\frac{\partial p_{r}}{\partial z}-\rho g\right]
$$

Where $k_{x}$ is xylem conductivity.

\section{Model for conservation of water inside of the root xylem}

We consider a unit length of the root and assume that there is balance between water flow through the root surface membrane and upward in the xylem tubes. Then mass conservation law yields [2][8][13],

$$
2 \pi a k_{r}\left(p-p_{r}\right)=\frac{\partial q}{\partial z} .
$$

Where $a$ is the radius of the root and $q$ is the longitudinal flux of water. Substituting the value of (4.4) in equation (5.1), then we get an ordinary differential equation for $p_{r}$ as a function of $z$, i.e.,

$$
2 \pi a k_{r}\left(p-p_{r}\right)=-k_{x} \frac{\partial^{2} p_{r}}{\partial z^{2}} .
$$

Which we can solve, subject to boundary conditions

$$
p_{r}=T \text { at } z=0, \text { and } \frac{\partial p_{r}}{\partial z}=0 \text { at } z=L .
$$

Where $T$ is the average pressure at the top of the root, i.e, at the root-shoot boundary and $L$ is the length of the root.

\section{Model for conservation of water inside of the root xylem if sur- rounding soil water pressure is constant}

Suppose the root surrounded by water in porous medium are at constant pressure, i.e., $\mathrm{p}=\mathrm{P}$, then wa- 
ter pressure equation in the root system is

$$
2 \pi a k_{r}\left(P-p_{r}\right)=-k_{x} \frac{\partial^{2} p_{r}}{\partial z^{2}} .
$$

Non-dimensionalise the equation (6.1) with

$$
z=L z^{*} \text { and } p_{r}=T p_{r}^{*}
$$

Where $z^{*}$ and $p_{r}^{*}$ are the dimensionless length and pressure respectively. The dimensionless equation (6.2) becomes with dropping $*$ becomes

$$
\kappa^{2}\left(P_{0}-p_{r}\right)=-\frac{\partial^{2} p_{r}}{\partial z^{2}}
$$

With dimensionless boundary condition (5.3) becomes

$$
p_{r}=1 \text { at } z=o \text { and } \frac{\partial p_{r}}{\partial z}=0 \text { at } z=1 .
$$

Where $P_{0}=\frac{P}{T}$ and $\kappa^{2}=\frac{2 \pi a k_{r} L^{2}}{k_{x}}$ are the dimensionless water uptake coefficients. Solution of equation (6.3) with boundary conditions $(6.4)$ is given by $\left.p_{r}=P_{0}+\frac{1}{2}\left[\left(P_{0}-1\right)\right] \tanh (\kappa)-P_{0}+1\right] e^{\kappa z}$

$$
+\frac{1}{2}\left[-\left(P_{0}-1\right) \tanh (\kappa)-P_{0}+1\right] e^{-\kappa z} .
$$

\section{Model for water flow in the surrounding of the root in the soil considering constant root in- ternal pressure as root surface boundary condition}

Water flow in soil given by equation (2.12) can be written in cylindrical radial co-ordinates form with boundary condition (3.7) is given by [14]

$$
\left(\phi_{l, s}-\phi_{l, r}\right) \frac{\partial S}{\partial t}=\frac{1}{r} \frac{\partial}{\partial r}\left(D(S) r \frac{\partial S}{\partial r}\right) .
$$

With boundary condition

$$
\begin{array}{r}
S=S_{\infty}, a \leq r \leq r_{\infty}, t=0 \\
D(S) \frac{\partial S}{\partial r}=k_{r}\left[p(S)-P_{r}\right] \text { at } r=a, \quad t>0 \\
S \rightarrow S_{\infty}, r \rightarrow r_{\infty}, t>0 .
\end{array}
$$

Where $P_{r}$ is the constant pressure inside the root and $D(S)=\frac{k_{s} \rho g}{\mu} \times \frac{(1-m)}{\alpha m} S^{1 / 2-1 / m}\left[\left(1-S^{1 / m}\right)^{-m}\right.$

$$
\left.+\left(1-S^{1 / m}\right)^{m}-2\right] .
$$

$$
p(S)=-\frac{\rho g}{\alpha}\left(S^{-1 / m}-1\right)^{1 / n} .
$$

We non-dimensionalise this model with choice of time, radius and pressure in new scales as follows

$t=[t] t^{*}=\frac{\left(\phi_{l, s}-\phi_{l, r}\right) \alpha m a^{2} \mu}{(1-m) k_{s} \rho g} t^{*}, r=a r^{*}, p=\frac{\rho g}{\alpha} p^{*}$.

And the dimensionless model with boundary condition becomes by dropping $*$

$$
\frac{\partial S}{\partial t}=\frac{1}{r} \frac{\partial}{\partial r}\left(D(S) r \frac{\partial S}{\partial r}\right) .
$$

$$
\begin{array}{r}
S=S_{\infty}, 1 \leq r \leq r_{\infty} / a, t=0 \\
D(S) \frac{\partial S}{\partial r}=\lambda_{w}\left[p(S)-P_{r}\right] \text { at } r=1, t>0 \\
S \rightarrow S_{\infty}, r \rightarrow \frac{r_{\infty}}{a}, t>0 .
\end{array}
$$

Where

$D(S)=S^{1 / 2-1 / m}\left[\left(1-S^{1 / m}\right)^{-m}+\left(1-S^{1 / m}\right)^{m}-2\right]$,

$$
p(S)=-\left(S^{-1 / m}-1\right)^{1 / n} \text {. }
$$

And dimensionless parameters are

$$
\lambda_{w}=\frac{k_{r} \rho g m a}{(1-m) K_{s}} \text { with } K_{s}=\frac{k_{s} \rho g}{\mu} \text { and } p_{r}=\frac{P_{r} \alpha}{\rho g} .
$$

We solve the equation (7.6) with boundary condition (7.7) by using Goodman integral transformation and obtain the solution in term of Bessel's function, use integral transform as follows [16]

$$
\psi=\int_{S}^{S_{\infty}} D(S) d S
$$

Then the above equation (7.6) with boundary condition is transform as

$$
\frac{1}{D(S)} \frac{\partial \psi}{\partial t}=\frac{1}{r} \frac{\partial}{\partial r}\left(r \frac{\partial \psi}{\partial r}\right)
$$

$$
\begin{array}{r}
\psi=0, \quad 1 \leq r \leq r_{\infty} / a, t=0, \\
\frac{\partial \psi}{\partial r}=\lambda_{w}\left[p(S)-P_{r}\right] \text { at } r=1, t>0, \\
\psi=o ; \text { at } r=\frac{r_{\infty}}{a}, t>0 .
\end{array}
$$

Solution of the equation (7.11) is of the form

$$
\psi(r, t)=\psi_{1}(r)+\psi_{2}(r, t) .
$$


Where

$$
\psi_{1}(r)=c_{1} \log r+c_{2} .
$$

Differentiating equation (7.14) we have

$$
\frac{\partial \psi}{\partial r}=\frac{c_{1}}{r} .
$$

Substitute the boundary conditions of system (7.12) into (7.15) and rearranging, yields, then we have

$$
\begin{gathered}
c_{1}=\lambda_{w}\left[p(S)-p_{r}\right] . \\
c_{2}=-\lambda_{w}\left[p(S)-p_{r}\right] \frac{\log r_{\infty}}{a} .
\end{gathered}
$$

Then the solution (7.14) is written as

$$
\begin{gathered}
\psi_{1}=\lambda_{w}\left[p(S)-p_{r}\right] \log r-\lambda_{w}\left[p(S)-p_{r}\right] \frac{\log r_{\infty}}{a} . \\
\psi_{1}=\lambda_{w}\left[p(S)-p_{r}\right] \log \frac{r}{r_{\infty} / a} .
\end{gathered}
$$

Assume that;

$$
\psi_{2}=R(r) r(t)
$$

Substitute equation (7.20) into equation (7.11) and using the method of separation of variables, then rearranging yields [3];

$$
\begin{gathered}
R(r)=c_{1} J_{0}(\lambda r)+c_{2} Y_{0}(\lambda r) . \\
r(t)=c_{3} e^{\lambda^{2} D(S) t} .
\end{gathered}
$$

Substitute value (7.19), (7.21) and (7.22) into equation (7.13) yields;

$$
\begin{aligned}
\psi(r, t)= & \left.\lambda_{w}\left[p(S)-p_{r}\right] \log \frac{r}{r_{\infty} / a}\right] \\
& +\left[c_{1} J_{0}(\lambda r)+c_{2} Y_{0}(\lambda r)\right] c_{3} e^{\lambda^{2} D(S) t} .
\end{aligned}
$$

To estimate the value of $(\lambda)$, substitute the boundary condition of system (7.12) into equation (7.23) and rearranging yields;

$\left.c_{2}=-c_{1} \frac{J_{0}\left(\lambda r_{\infty} / a\right)}{Y_{0}\left(\lambda r_{\infty} / a\right.}\right)$,

$\frac{J_{0}(\lambda)}{Y_{0}(\lambda)}=\frac{J_{0}\left(\lambda r_{\infty} / a\right)}{Y_{0}\left(\lambda r_{\infty} / a\right)} \Rightarrow \frac{J_{0}(\lambda)}{J_{0}\left(\lambda r_{\infty} / a\right)}=\frac{Y_{0}(\lambda)}{Y_{0}\left(\lambda r_{\infty} / a\right)}=\sigma$,

$$
J_{0}\left(\lambda_{n}\right) Y_{0}\left(\lambda_{n} r_{\infty / a}\right)-J_{0}\left(\lambda_{n} r_{\lambda / a}\right) Y_{0}\left(\lambda_{n}\right)=0 .
$$

The values of $\left(\lambda_{n}\right)$ represent the roots of equation (7.24), where $(n=1,2,3, \ldots, \infty)$. Assume

$U_{0}\left(\lambda_{n} r\right)=J_{0}\left(\lambda_{n} r\right) Y_{0}\left(\lambda_{n} r_{\infty} / a\right)-J_{0}\left(\lambda_{n} r_{\infty} / a\right) Y_{0}\left(\lambda_{n} r\right)$.

Substitute the initial conduction of system equation (7.12) into equation (7.23) and use equation (7.25), then rearranging, yields;

$c_{1} c_{2} U_{0}\left(\lambda_{n} r\right)=Y_{0}\left(\lambda_{n} r_{\infty} / a\right)\left[-\lambda_{w}\left[p(S)-p_{r}\right] \log \frac{r}{r_{\infty} / a}\right]$, $f(r)=Y_{0}\left(\lambda_{n} r_{\infty} / a\right)\left[-\lambda_{w}\left[p(S)-p_{r}\right] \log \frac{r}{r_{\infty} / a}\right]$.

Hence

$$
\begin{aligned}
f(r)=c_{1} U_{0}\left(\lambda_{1} r\right) & +c_{2} U_{0}\left(\lambda_{2} r\right)+c_{3} U_{0}\left(\lambda_{3} r\right)+\ldots \\
& =\sum_{n=1}^{\infty} c_{n} U_{0}\left(\lambda_{n} r\right) .
\end{aligned}
$$

Multiply equation (7.26) by $\left[r U_{0}\left(\lambda_{m} r\right)\right]$ and integrate the result over the integral $\left[1, r_{\infty} / a\right]$ with the assumption that the integral of the infinite sum is equivalent to the sum of the integration we have $\int_{1}^{r_{\infty} / a} r f(r) U_{0}\left(\lambda_{m} r\right) d r$

$$
=\sum_{n=1}^{\infty} \int_{1}^{r_{\infty} / a} r U_{0}\left(\lambda_{m} r\right) U_{0}\left(\lambda_{n} r\right) d r .
$$

All the terms on the right hand side of equation (7.27) equals zero, except when $(\mathrm{m}=\mathrm{n})$, hence;

$$
c_{n}=\frac{\int_{1}^{r_{\infty} / a} r f(r) U_{0}\left(\lambda_{n} r\right) d r}{\int_{1}^{r_{\infty} / a} r U_{0}^{2}\left(\lambda_{n} r\right) d r} .
$$

The denominator of equation (7.28) is estimated as follows, [18]

$$
\int_{1}^{r_{\infty} / a} r U_{0}^{2}\left(\lambda_{n} r\right) d r=\frac{2\left[J_{0}^{2}\left(\lambda_{n}\right)-J_{0}^{2}\left(\lambda_{n} r_{\infty} / a\right)\right]}{\pi^{2} \lambda_{n} 2 J_{0}^{2}\left(\lambda_{n}\right)} .
$$

To estimate the numerator of equation (7.28), the following are used, [6]

$$
\begin{aligned}
& \int_{1}^{r_{\infty} / a} r U_{0}\left(\lambda_{n} r\right) d r=\frac{2\left[J_{0}\left(\lambda_{n}\right)-J_{0}\left(\lambda_{n} r_{\infty} / a\right)\right]}{\pi \lambda_{n}^{2} J_{0}\left(\lambda_{n}\right)}, \\
& \int_{1}^{r_{\infty} / a} r \ln (r) U_{0}\left(\lambda_{n} r\right) d r=\frac{2\left[J_{0}\left(\lambda_{n}\right)\right] \ln \left(r_{\infty} / a\right)}{\pi} \lambda_{n}^{2} J_{0}\left(\lambda_{n}\right) . \\
& \text { Hence } \\
& \int_{1}^{r_{\infty} / a} r f(r) U_{0}\left(\lambda_{n} r\right) d r \\
& \qquad \quad=\frac{2}{\pi \lambda_{n}^{2}} \frac{\left[Y_{0}\left(\lambda_{n} r_{\infty} / a\right)\right]\left[-J_{0}\left(\lambda_{n} r_{\infty} / a\right)\right]}{J_{0}\left(\lambda_{n}\right)}
\end{aligned}
$$

Substituting equation (7.29) and (7.30) into (7.28) yields:

$$
c_{n}=\frac{\pi\left[Y_{0}\left(\lambda_{n} r_{\infty} / a\right)\right]\left[-J_{0}\left(\lambda_{n} r_{\infty} / a\right)\right]\left[J_{0}\left(\lambda_{n}\right)\right]}{\left[J_{0}^{2}\left(\lambda_{n}\right)-\lambda_{0}^{2}\left(\lambda_{n} r_{\infty} / a\right)\right]} .
$$

Substitute equation (7.31) into (7.23) and rearranging yields;

$\psi(r, t)=\left[\lambda_{w}\left[p(S)-p_{r}\right] \log \frac{r}{r_{\infty} / a}\right]$

$$
-\sum_{n=1}^{\infty} \frac{\pi\left[J_{0}\left(\lambda_{n} r_{\infty} / a\right)\right]\left[J_{o}\left(\lambda_{n}\right)\right]}{\left[J_{0}^{2}\left(\lambda_{n}\right)-J_{0}^{2}\left(\lambda_{n} r_{\infty} / a\right)\right]} U_{0}\left(\lambda_{n} r\right) e^{\lambda^{2} D(S) t} .
$$


Hence solution of equation (7.6) is given by

$$
\begin{aligned}
& \int_{S}^{S_{\infty}} D(S) d S=\left[\lambda_{w}\left[p(S)-p_{r}\right] \log \frac{r}{r_{\infty} / a}\right] \\
& -\sum_{n=1}^{\infty} \frac{\pi\left[J_{0}\left(\lambda_{n} r_{\infty} / a\right)\right]\left[J_{o}\left(\lambda_{n}\right)\right]}{\left[J_{0}^{2}\left(\lambda_{n}\right)-J_{0}^{2}\left(\lambda_{n} r_{\infty} / a\right)\right]} U_{0}\left(\lambda_{n} r\right) e^{\lambda^{2} D(S) t} .
\end{aligned}
$$

Time independent form of equation (7.1) can also written in term of relative water saturation in the soil [15]. The equation for conservation of water in the soil is thus

$$
\phi \frac{\partial S}{\partial t}+\nabla \cdot \mathbf{u}=-F_{w} .
$$

Where $\phi$ is the (constant) porosity of the soil, $S$ is the relative water saturation in the soil i.e., $S=\phi_{l} / \phi$, where $\phi_{l}$ is the volumetric water fraction, $\mathbf{u}$ is the volume flux of water, i.e., Darcy flux and $F_{w}$ is the uptake of water by plant roots. Consider value of $\mathbf{u}$ given in equation (2.7) with equation (2.10). Also the water pressure in the soil pores can be linked to the relative water saturation via suction characteristic [10],

$$
p_{a}-p=p_{c} f(S), f(S)=\left(S^{-1 / m}-1\right)^{1-m} .
$$

Where $p_{a}$ is atmospheric pressure, and $p_{c}$ is a characteristic suction pressure. It is common to take $p_{a}=0$. Then the Darcy-Richards equation in terms of relative water saturation only, it is written as

$$
\phi \frac{\partial S}{\partial t}=\nabla \cdot\left[D_{o} D(S) \nabla S-K_{s} K(S) \hat{\mathbf{k}}-F_{w}\right] .
$$

Where the water diffusivity in the soil is $D_{0} D(S)=$ $\frac{k}{\mu} \frac{\partial p}{\partial S}, D_{0}=\frac{p_{c} k_{s}}{\mu}\left(\frac{1-m}{m}\right)$ and

$$
D(S)=S^{1 / 2-1 / m}\left[\left(1-S^{1 / m}\right)^{-m}+\left(1-S^{1 / m}\right)^{m}-2\right] .
$$

Also equation (2.8) can be written as follows

$$
K_{s}=\frac{\rho g k_{s}}{\mu} .
$$

Equation (7.36) are studied with boundary condition at soil surface and at the base of soil layer. If amount of water is flowing due to rainfall, then we take boundary

$$
-D_{0} D(S) \frac{\partial S}{\partial z}=q_{s}, \quad \text { at } z=0 .
$$

Where $q_{s}$ is the volume flux of water per unit soil surface area per unit time. If surface pounding occur, then the boundary condition will be $S=1$ at $z=0$. The boundary condition at base of soil layer is written as

$$
-D_{0} D(S) \frac{\partial S}{\partial z}+K_{s} K(S)=0, \text { at } z=l_{w} .
$$

The model for water uptake of a single cylindrical root in cylindrical radial co-ordinates is written as

$$
\phi \frac{\partial S}{\partial r}=\frac{1}{r} \frac{\partial}{\partial r}\left(D_{0} D(S) r \frac{\partial S}{\partial r}\right) .
$$

With boundary conditions

$$
\begin{array}{r}
D_{0} D(S) \frac{\partial S}{\partial r}=k_{r}\left[p(S)-p_{r}\right] \text { at } r=a, \\
\frac{\partial S}{\partial r}=0 \text { at } r=a_{\text {int }} .
\end{array}
$$

Where

$$
p(S)=-p_{c}\left(S^{-1 / m}-1\right)^{1-m},
$$

$a$ is the radius of the root, $a_{i n t}$ is a measure of inter branch distance, $k_{r}$ is root radial conductivity and $p_{r}$ is the root internal pressure, $P \leq p_{r} \leq p(S)$.

We take the far-field soil water content, i.e., effective water saturation is $S_{\infty}$ which is constant. Then the far-field boundary condition is given by

$S \rightarrow \infty$ as $r \rightarrow \infty$.

Non-dimensionalizing this model with $r=a, t=$ $\phi a^{2} / D_{0}$ and $p_{r}=|P|$,

$$
\frac{\partial S}{\partial t}=\frac{1}{r} \frac{\partial}{\partial r}\left[r D(S) \frac{\partial S}{\partial r}\right] .
$$

With boundary conditions

$$
\begin{array}{r}
D(S) \frac{\partial S}{\partial r}=\lambda\left[-\varepsilon_{0} f(S)-p_{r}\right] \text { at } r=1, \\
\frac{\partial S}{\partial r}=0 \text { at } r=a_{\text {int }} .
\end{array}
$$

Where $\lambda=k_{r} a|P| / D_{0}$ and $\varepsilon=p_{c} /|P|$.

The far-filed boundary condition is still

$S \rightarrow \infty$ as $r \rightarrow \infty$.

Solution of equation (7.44) is given by [13] for outer region far away from the root

$$
S=S_{\infty}+s=S_{\infty}+B E_{1}\left(\frac{r^{2}}{4 t D\left(S_{\infty}\right)}\right) .
$$

Where $S(r, t)=S_{\infty}+s(r, t)$. For inner region near the root solution is given by

$$
S=S_{\infty}+s_{1}+\frac{\lambda_{w}\left[p\left(S_{\infty}-p_{r}\right)\right]}{D\left(S_{\infty}\right)} \ln r .
$$


Where $s_{1}=-\frac{\lambda_{w}\left[p\left(S_{\infty}-p_{r}\right)\right]}{2 D\left(S_{\infty}\right)} \ln \left[4 e^{-\gamma} D\left(S_{\infty}\right) t+1\right]$.

Also we have the dimensional expression for water uptake by root from soil given in equation (7.34) which is written as [7],

$$
F_{w}=2 \phi a l_{d} k_{r}\left[-p_{c} f(S)-p_{r}\right] .
$$

Where $l_{d}$ is the root length density.

\section{Discussion}

Henry Darcy studied the flow of water in porous soil experimentally. For water flow in unsaturated soil, the effective water saturation is defined in terms of saturation soil contempt. Van Genuchten write conductivity, in terms of effective water saturation. Minimal moisture contempt is considerable if soil is unsaturated and if saturation reaches in short time, then problem is studied as time dependent initial and boundary value problem. If soil is nearly about saturated then the saturation soil contempt is nearly equal to porosity. Then the effective water saturation is equivalent to relative water saturation and problem is studied as time independent boundary value problem. Formula of effective water saturation reduces to relative water saturation just taking $\phi_{l, r} \approx o$. The boundary condition for water flow, from soil to xylem is the water pressure in the root surface which contain cortex tissues, which work as semipermeable membrane having inner and outer radius. Therefore the water pressure at the inner radius of cylindrical root is the water pressure for flow of water from root to leaves through the stem. The equation of water flow through the stem is same as Poiseuille flow. We considered root as cylinder and calculated conservation of water in the root by flow of water through root surface considering water pressure in the soil is constant. Also we derived the water profile surrounding root surface in porous soil considering root having constant internal pressure as boundary condition.

\section{References}

[1] Bear.J., Hydraulics of Groundwater, McGrawHiil, 1959.

[2] Chu JiaQiang,Jiao WeiPing and JianJun, Mathematical modelling study for water uptake of steadily growing plant root, sci china Ser G-Phy Mech Astron vol 51 no 2, 2008, 184-205.
[3] Dr.Mishaal Abdulameer Abdulkareem, Analytical Solution of Transient Heat Conduction through a Hollow Cylindrical Thermal Insulation Material of a Temperature Dependant Thermal Conductivity, Journal of Engineering 11(19), 2013, 1483-1503.

[4] F.J.Molz, Models of water transport in the soilplant system, Water Resources Research, 17(5), 1981, 1245-1260 .

[5] Gangurde AB and Boraste SS, Perliminary evalution of bauhinia racemosa lam caesalpinaceae seed mucilage as tablet binder, Int J Pharm 2(1), 80-83, 2012.

[6] H.S.Carslaw and J.C.Jaeger, Conduction of heat in solid, Oxford University press 1959.

[7] J.Crank, The Mathematics of Diffusion, Oxford University Press, 1975.

[8] J.J.Landsberg and N.D.Fowkes, Water movement through plant roots, Annals of Botany 42, 1978, 493-508.

[9] Melvin T.Tyree, Shudong Yang, Pierre Cruiziat, and Bronwen Sinclair, Novel Methods of Measuring Hydralic conductivity of tree root Systems and Interpretation using AMAIZED, plant physiol, 104, 1994, 189-199.

[10] M.TH.Van Genuchten, A closed-form equation for predicting the hydraulic conductivity of unsaturated soils, soil science Society of America journal, 44, 1980, 892-898.

[11] Prasad, Rama, A linear root water uptake model, In: Journal of Hydrology, 99 (3-4), 1988, 297306.

[12] Ritchie J.T., A user-orientated model of the soil water balance in wheat, What growth and modeling, New York, 86, 1985, 293-305.

[13] Roose T., Mathematical model of plant nutrient uptake, Doctor Dissertation.Oxford Linacre College, University of Oxford, 2000.

[14] T.Roose A.C Fowler, A mathematical model for water and nutrient uptake by plant root systems, Journal of Theoretical Biology, 228, 2004, 173184. 
[15] T.Roose, A.C.Fowler, A mathematical model for water and nutrient uptake by plant root systems, Journal of Theoretical Biology 228, 2004, 155171.

[16] T.R. Goodman, "Application of Integral Methods to Transient Nonlinear Heat Transfer," in Advances in Heat Transfer 1, edited by Academic press, 1964,493-508.

[17] Van Dam J.C., Huygen J., Wesseling J.G., Feddes R.A., Kabat P.,van Valsum P.E.V., Groenendijk P. and van Diepen C.A., Theory of SWAP, Version 2.0. Simulation of Water Flow, Solute Transport and Plant Growth in the SoilWater-Atmosphere- Plant Environment, Technical Document 45, Wageningen 1997.

[18] Vineet Kumar, Sudesh Kumar Yadav, Synthesis of variable of shaped goil nanoparticles in one solution using leaf extract of Bauhinia variegata $l$, Digest Journal of Nanomaterials and Biostructures, 6(4), 2011, 1685-1693. 